\title{
Comparative Study between Traditional Grammar and Modern Linguistics
}

\author{
Jian $\mathrm{Li}^{*}$ and Qing Ming $\mathrm{Li}$ \\ Faculty of Humanities and Foreign Language, Xi'an University of Technology, Shaanxi Xi'an, China \\ ccyxz@xaut.edu.cn,nocc@163.com
}

Keywords: Traditional grammar; Modern linguistics; Descriptive grammar; Difference; relationship

\begin{abstract}
The paper gives a brief introduction on Traditional grammar and Modern linguistics, and mainly analyses their similarities and differences. The standard the Traditional grammar established is according to the language used by the writers of previous centuries and also gave classical examples. Modern linguistics is a science of linguistic study, which starts from descriptive grammar, structure grammar, functional grammar, transformational-generative grammar (case grammar) and many other grammars. It points out that though they are quite different, Traditional grammar is the base from which Modern linguistics is derived.
\end{abstract}

\section{Introduction}

Language changes, from generation to generation, at levels of sound, form and meaning. Thus grammar, as the method of analyzing these changes, altered accordingly. Now we have mainly two branches, the Traditional grammar and Modern linguistics. It is necessary to give a comment on their significance as well as weaknesses, and their differences as well as similarities.

\section{Traditional Grammar}

As the most ancient grammar, Traditional grammar has its origins in the 15th century B.C., with Plato and Aristotle in Greece and a Sanskrit scholar named Panini in India. Various Romans and early-Christian-era writers also made contribution to the Traditional grammar, but the most influential of the Traditional grammarians began writing in the 18th century, about the time when English was beginning to be taken seriously as a separate language and not as merely another vernacular [1]. The features of Traditional grammar can be illustrated according to following aspects.

First, one of the main features of grammar is its usually based on meaning. According to Traditional grammar, a sentence is a group of words that express a complete idea. Traditional Grammar often analyses it from meaning, from meaning to form; from the viewpoint of language teaching, Traditional grammar doesn't give a systematic description of linguistic phenomenon. It often gives description at surface level and often analyzes a sentence in isolation not at a discourse level [2]. And sometimes, it even without description level, so it doesn't provide the teacher with a satisfactory description of language he is teaching, and not provide for the student sufficient description of language he need to learn. Traditional grammar usually describes the written language, without considering and studying the oral language. And also, it confuses the written with the oral form, but as we know, the system of oral language differs in some degree to written language. So Traditional Grammar cannot have the students acquire the mechanism of oral communication. And Traditional grammar gives a predominant place to morphology and syntax, the treatment of lexis and phonology is very often inadequate in Traditional Grammar. Despite its disadvantages, Traditional Grammar is of great value to language teaching, school grammar, and a great many people still believe that it is a functional, elegant, time-honored way of teach people what they should know about language [3]. 


\section{Modern Linguistics}

Modern Linguistics began form the Swiss Linguist Ferdinanel de Saussure (1857-1913), who is often described as 'father of modern linguistics' and 'a master of a discipline which be made 'modern' (Culler 1976:7). Modern linguistics is a science of linguistic study. According to Modern Linguistics, language is a system and grammar is regarded as a systematic description of a certain language, either written or oral [4]. Grammar also refers to distributional analysis of surface structure elements according to distributional criteria. Also, phonetics, phonological and semantic components are considered in modern grammar [5]. Generally speaking, modern grammar is currently evaluated on the basis of applicability, simplicity, completeness, explicitness, and lack of contradiction. Modern grammar starts from descriptive grammar, structure grammar, functional grammar, transformational-generative grammar (case grammar) and many other grammars.

Descriptive Grammar. Descriptive grammar describes how a language is actually spoken and written and does not describe how a language ought to be spoken or written [6]. According to descriptive grammar, it states that speech is the basic form of language, and there is a difference between spoken and written language. Fries is a distinguished grammarian, his work 'American English Grammar' is a famous work. According to him, all words are classified into two parts: content words and functional words, not ten different parts of speech as in Traditional grammar. The content words refer to those words which have inflection and which have lexical meaning, such as noun, verb, adjective ect. Functional words are those words which place on important part in formulating structures, determines, subordinate conjunctions, auxiliaries and emphatic words.

Structural Grammar. Structural grammar is quite different form the Traditional Grammar. Instead if focusing on the individual word and its notional meaning or its part-of-speech function in the sentence, Structural grammar focuses on cluster of structures - sounds, forms, word groups, phrases - working from smaller to larger units. Structural grammar does not ignore semantic meaning (although some of its earlier advocates tried to do so.), but it tends to emphasize syntactic over semantic meaning. That is, Structural grammar analyzes the meaning carried by the syntactic patterns that morphemes and words make with each other, patterns like those formed by plural morphemes, modifier-verb or modifier-adjective connections, subject-predicate connections, and so on.

Besides the general emphasis on morphology and syntax, Structural grammar developed three particularly useful analytical techniques: test frames, immediate constituent analysis, and sentence formulas. Test frames especially have been helpful in teaching grammar in the schools [7].

The disadvantages of Structural grammar are as following.

It presents an incomplete description of the grammatical system of language, and does not provide the rules needed to construct an infinite range of grammaticality.

It attaches excessive weight to morphological and morph-phonological rules, but semantic relations received slight attention, it is the same with Traditional grammar.

It describes the surface structure of sentences and mis-making a number of deep generalizations.

Structural grammar gives a criterion to determine grammaticality and degree of grammaticality of sentences. And it does not provide sufficient explanation to guarantee clear understanding and correct usage. This may lead learners to make errors.

It excludes the treatment of meaning, but any grammatical analysis will be of no use if meaning is not taken into consideration.

It doesn't provide satisfactory basis for another two important areas: constructive analysis and translation in applied linguistics.

Transformational-generative Grammar. Transformational-generative grammar, TG grammar, is developed by Norman, Chomsky. It appeared in 1957 when a revolution occurred in linguistics. According to some linguistics, TG grammar is a synthesis of contribution of Traditional grammar and Structural grammar. As far as Structural grammar is concerned, Chomsky reconstruct ICA (Immediate Constituent Analysis) as a first stage of his grammar, but he went much further and satisfied the demands of precision in the formalization of his undergone several stages: classical theory, standard theory, extended theory, and revised extended theory [8]. 
In the first stage, representative work, 'Syntactic Structure', it deals with an infinite set of sentences produced by context free structure. If forms the basic transformational rules. In the second stage, with the representative work 'Aspects of the Theory of Syntax', the original syntactic theory is extended to a general theory of grammar which includes phonology and semantics. The basis of the syntax is the deep structure, and the surface structure phenomenon, such as intonation, word order and themes-rheme. In the stage of extended theory, the focus has been transformed from individual grammar o universal grammar. In this stage, all transformational rules are reduced to only one rule, that it move 2. Also in this stage, the universal formulation of constraint is developed. Hence, the advantages of TG grammar are: first, it actually combines syntax phonology, lexicon, and semantics. So, it gives an overall conception of the system language. And this system is more accurate and more complete that other grammatical model. Second, TG grammar gives a more economical and systematic description of language, it provides a system of rules which permits the generation of an infinite number of grammatical sentences. Unlike Traditional grammar, rules stated in TG grammar are very clear and formally explicit. Third, TG grammar shows us very clearly that it processes a great generalizing power. It is also able to clarify underlying structures and regularities, which have been ignored by grammarians of Traditional grammar and Structural grammar. Fourth, TG grammar admits the existence of linguistic universals and analysis between languages at the level of deep structure. As for language universal, Traditional grammar admits the existence of language universals, but Structural grammar objects this view. According to Structural grammar, each language presents an individual structure. But TG grammar admits that descriptions of all languages have the same general form and the same type of rules. This refers to the former universals. And they also present common categories and deep structures and this refers to the substantive universals, and the last advantage of TG grammar is that it can characterize notions of grammaticality and degree of grammaticality which are indispensable in the area of evaluation, testing and error analysis.

Functional Grammar. Functional Grammar was created by M.A.K. Halliday. It was called systematic grammar in 1950. In Functional grammar, meaning is taken as the aim of what's the speaker wants the hearer to understand [9]. Here, the meaning of a sentence is equated with its function. The aim if Functional grammar is to study the range of relevant choices of meaning and wording. And one important implication of the functional view of language is its context. That is to say, Functional grammar takes context into consideration, and it takes linguistics toward sociology. That is the systematic study of the relevant features in the culture and society, which form the context in which language used. According to Functional grammar, all words can be divided into open set and closed set. Open set are noun, verb adjective and adverb; they are lexical word or content word. Close set are also grammatical functioned words such as Pron, Conj, Prep, Article. In Functional grammar, group and phrase are two different concepts. Group is the extension of words, while phrase is the compression of clause. Phrase exclusively refers to P.P; also there are structured and functional labels in Functional grammar. Structural labels refer $t$ the nature of structure of elements, while functional labels refer to the syntactic function of clauses.

\section{The Differences between Modern Linguistics and Traditional Grammar}

Linguistics is descriptive not prescriptive. Most modern linguistics is descriptive, because it attempts to describe what people actually say, not what people should say [10]. It describes language in all its aspects, but does not prescribe rules of 'correctness'. This is in contrast with the study of language in previous centuries. It was mostly prescriptive. Traditional grammars told people how to use a language. Modern linguists, however, do not believe that there is an absolute standard of correctness concerning language use which linguists or school teachers should view as their duty to maintain. Instead, they would prefer to be observers and recorders of facts, but not judges. They believe that whatever occurs in natural speech ( hesitation, incomplete utterance, misunderstanding, etc. ) should be describe in their analysis. They might recognize that one type of speech appears to be more socially acceptable than others because of the influence of fashion. But this will not make them 
think that socially acceptable variety can replace all the other varieties, or the old words are always better than new ones or vice visa. They will regard the changes in language and language uses as the result of a natural and continuous precess, but not something to be feared. Language changes should be observed and described. However, this does not deny that languages have rules. They obviously do or we would not understand each other. On the other hand, no single rule or expression is necessarily there forever.

Linguistics regards the spoken language as primary, not the written. In the past, grammarians have overstressed the importance of the written word, partly because of its permanence. It was difficult to cope with fleeting utterances before the invention of sound recording. The traditional classical education was also partly to blame. People insisted on molding language in accordance with the usage of the 'best authors' of classical times and these authors existed only in written form.

As a matter of fact, however, whether we think of the history of human speech in general of if the linguistic experience of the individual speaker, spoken language is the primary phenomenon, and writing is only a more or less imperfect reflection of it. We all learn to understand speech before we learn to read, and to speak before we learn to write. We all hear more language than we read and speak a great deal more than we write. Spoken language is ordinarily more flexible tan written language; it leads the way in linguistic development, while written language follows at a greater or less interval.

Spoken language is considered as the primary medium for several reasons. Spoken language is prior to written language historically. In other words, it existed long, long before written systems came into being. Even today many well-developed languages do not have a written system yet. Genetically, children always learn to speak before they learn to write. Blind children have no difficulty in learning to speak but deaf children have great difficulty in learning to read. This shows that the channel of sight is not as important as the channel of sound in learning a language.

However, this is not to deny the importance of written language, which has its own advantages that spoken language does not have. First, with written language, messages can be carried through space. Human voice is effective only within earshot. With the help of written language, we can send and receive messages across vast spaces. Secondly, with written language, messages can be carried through time. The spoken word 'dies' immediately, but a written message can be transmitted far beyond the moment of production ----- often from generation to generation and from one culture to another. Thirdly, oral message are subject to distortion, either unintentional (when due to misunderstanding for example) or otherwise. Written messages, on the other hand, remain exactly the same whether read a thousand years later or ten thousand miles away.

Spoken utterances share many common features with written sentences, but they also exhibit considerable differences. Therefore linguists believe spoken forms and written forms belong to different systems though they may overlap. The systems must be analyzed separately: the spoken first, then the written.

Linguistics differs from traditional grammar in that it does not force languages into a Latin-based framework. In the past, many traditional textbooks have assumed unquestionably that Latin provides a universal framework into which all languages fit, and countless school children have been confused by meaningless attempts to force English into foreign patterns. It is sometimes claimed, for example, that a phrase such as for John is in the 'dative case'. But this is blatantly untrue, since English does not have a Latin-type case system. At other times, the influence of the Latin framework is more subtle, and so more misleading. Many people have wrongly come to regard certain Latin categories as being 'natural' ones. For example, it is commonly assumed that the Latin tense divisions of past, present and future are inevitable. Yet one frequently meets languages which do not make this neat threefold distinction. In some languages, it is more important to express the duration of an action ----- whether it is a single act or a continuing process than to locate the action in time.

In addition, judgments on certain constructions often turn out to have a Latin origin. For example, people frequently argue that 'good English' avoids 'split infinitives' as in the phrase to humbly apologize, where the infinitive to apologize is 'split' by humbly. The idea that a split infinitive is wrong is based on Latin. Purists insist that, because a Latin infinitive is only one word, its English 
equivalent must be as near to one word as possible. To linguists, it is unthinkable to judge one language by the standards of another. They are opposed to the notion that any one language can provide an adequate framework for all the others. They are trying to set up a universal framework, but that will be based on the features shared by most of the languages used by mankind.

\section{Summary}

Although there are great differences between Modern linguistic and Traditional grammar, much of the work done descriptively, rather than prescriptively, in contemporary grammatical analysis was couched in Traditional grammar language by scholar like Otto Jespersen, W. Nelson Francis and Henrik Poutsma. To understand any of the modern grammars, and to understand virtually all discussion about writing or literature at the level of stylistic analysis, one must have an understanding of the terminology drawn from Traditional grammar, if not of the whole system.

\section{Acknowledgment}

This topic of research is supported by Philosophy and Social Science Research Project Plan of shaanxi Province (No. 13L048) and Major Theoretical and Practice Issues Research Projects of Shaanxi Social Science Association (No. 2013C104).

\section{References}

[1] Mojtaba Rajabi, Khadijeh Aghaei, Moses Samuel, Revisiting Challenges of Traditional \& Transformational Grammar in ELT: A Sign-based Approach, Procedia - Social and Behavioral Sciences. 1118 (2014) 378-382.

[2] A McMahon, Historical Linguistics: Overview, International Encyclopedia of the Social \& Behavioral Sciences. 35(2001) 6748-6753.

[3] Azer, B, Understanding and Using English Grammar, Third Edition, Longman, 1998.

[4] John E. Joseph, Modern linguistics in post-modern perspective, Language \& Communication. 12 (1992) 165-179.

[5] Noguchi, R, Grammar and the teaching of writing: limits and possibilities, Urbana: NCTE, 1991.

[6] Peter Schmitter, The theory of word formation in early semasiology: A blank spot on the map of 19th century linguistics, Language Sciences. 30 (2008) 575-696.

[7] Joseph L. Subbiondo, An introduction to grammar: traditional, structural, transformational, Lingua. 43(1977), 401,404-405.

[8] Frits Stuurman, English traditional grammars: An international perspective, Lingua. 90(1993) 271-278.

[9] Quirk R, Greenbaum S, Leech G and Svartik J, A Comprehensive Grammar of the English Language. London: Longman, 1985.

[10] Jason Moore, Mary Schleppegrell, Using a functional linguistics metalanguage to support academic language development in the English Language Arts, Linguistics and Education. 26(2014) 92-105. 